\title{
A multicentre, randomised, double-blind, placebo-controlled trial with the interleukin-1 receptor antagonist anakinra in patients with systemic-onset juvenile idiopathic arthritis (ANAJIS trial)
}

\author{
Pierre Quartier, ${ }^{1}$ Florence Allantaz, ${ }^{2}$ Rolando Cimaz, ${ }^{3}$ Pascal Pillet, ${ }^{4}$ Claude Messiaen, ${ }^{1}$ \\ Christophe Bardin, ${ }^{5}$ Xavier Bossuyt, ${ }^{6}$ Anne Boutten, ${ }^{7}$ Jacques Bienvenu, ${ }^{8}$ \\ Agnes Duquesne, ${ }^{3}$ Olivier Richer, ${ }^{4}$ Damien Chaussabel, ${ }^{2}$ Agnes Mogenet, ${ }^{1}$ \\ Jacques Banchereau, 2,9,10 Jean-Marc Treluyer, ${ }^{1}$ Paul Landais, ${ }^{1}$ Virginia Pascual ${ }^{2}$
}

\begin{abstract}
- Additional data
(supplementary tables and

figures) are published online

only. To view these files please

visit the journal online at (http://

ard.bmj.com).

'Université Paris-Descartes and Hôpital Necker-Enfants

Malades, Assistance Publique

Hôpitaux de Paris, Paris, France

${ }^{2}$ Baylor Institute for Immunology

Research, Dallas, Texas, USA

${ }^{3}$ Hôpital Femme-Mère-Enfant,

Lyon, France

${ }^{4}$ Hôpital Pellegrin Enfants,

Bordeaux, France

${ }^{5}$ Hôpital Hôtel Dieu, Assistance

Publique Hôpitaux de Paris,

Paris, France

6University of Leuven, Leuven,

Belgium

${ }^{7}$ Hôpital Xavier Bichat,

Assistance Publique Hôpitaux

de Paris, Paris, France

8Université Lyon Sud, Lyon,

France

${ }^{9}$ INSERM Unit 899, Dallas,

Texas, USA

${ }^{10}$ Mount Sinai School of

Medicine, New York, New York, USA
\end{abstract}

\section{Correspondence to}

Dr Pierre Quartier, Unite

d'Immuno-Hematologie et

Rhumatologie Pediatriques,

Hopital Necker-Enfants

Malades, 149 rue de Sevres 75

015 Paris, France;

pierre.quartier@nck.aphp.fr

PL and VP contributed equally to this work.

Accepted 11 November 2010 Published Online First 20 December 2010

\section{UNLOCKID}

This paper is freely available online under the BMJ Journals unlocked scheme, see http:// ard.bmj.com/info/unlocked.dtl

\begin{abstract}
Objectives To assess the efficacy of the interleukin 1 receptor antagonist anakinra in systemic-onset juvenile idiopathic arthritis (SJIA).

Methods A multicentre, randomised, double-blind, placebo-controlled trial was conducted. The primary objective was to compare the efficacy of a 1-month treatment with anakinra $(2 \mathrm{mg} / \mathrm{kg}$ subcutaneous daily, maximum $100 \mathrm{mg}$ ) with a placebo between two groups each with 12 patients with SJIA. Response was defined by a 30\% improvement of the paediatric American College of Rheumatology criteria for JIA, resolution of systemic symptoms and a decrease of at least $50 \%$ of both C-reactive protein and erythrocyte sedimentation rate compared with baseline. After month 1 (M1), patients taking placebo were switched to anakinra. Secondary objectives included tolerance and efficacy assessment for 12 months, and analyses of treatment effect on blood gene expression profiling.
\end{abstract}

Results At M1, 8/12 responders were receiving anakinra and 1 responder receiving placebo $(p=0.003)$. Ten patients from the placebo group switched to anakinra; nine were responders at $M 2$. Between $M 1$ and $M 12$, six patients stopped treatment owing to an adverse event $(n=2)$, lack of efficacy $(n=2)$ or a disease flare $(n=2)$. Blood gene expression profiling at enrolment and at 6 months' follow-up showed one set of dysregulated genes that reverted to normal values in the clinical responders and a different set, including interferon (IFN)-inducible genes, that was induced by anakinra.

Conclusions Anakinra treatment is effective in SJIA, at least in the short term. It is associated with normalisation of blood gene expression profiles in clinical responders and induces a de novo IFN signature.

Trial Registration Number: NCT00339157.

\section{INTRODUCTION}

Systemic-onset juvenile idiopathic arthritis (SJIA) is a disease of unknown aetiology characterised by arthritis and systemic symptoms starting before the age of $16 .{ }^{1}$ The most characteristic feature at onset is spiking fever, which is often associated with an evanescent skin rash, hepatosplenomegaly and pericarditis. Non-steroidal anti-inflammatory drugs, corticosteroids, methotrexate and antitumour necrosis factor (anti-TNF) agents are often only partially effective. ${ }^{2} 3$ The anti-interleukin
6 receptor antagonist tocilizumab was effective in one randomised withdrawal trial. ${ }^{4}$ Patients with a persistently systematically active course 6 months after disease onset, while receiving corticosteroid treatment, are at high risk of longlasting, nonremitting or polycyclic disease..$^{5}$

Interleukin 1 (IL-1) has an important role in SJIA. In particular, serum samples from patients with SJIA induce IL-1 $\beta$ transcription on healthy peripheral blood mononuclear cells (PBMCs), and treatment with the IL-1 receptor antagonist (IL-1Ra) anakinra leads to the normalisation of a disease-specific gene expression profile. ${ }^{6} 7$ Non-controlled pilot studies provide evidence of dramatic, quick responses to anakinra in a subgroup of patients with SJIA. ${ }^{6-10}$ However, some patients may have a self-remitting course, and no placebo-controlled studies have been published to evaluate the effects of IL-1 blockade.

We therefore designed a trial aiming at assessing the efficacy of anakinra treatment, and its effect on blood gene expression profiling, in patients with SJIA displaying active systemic disease despite corticosteroid treatment.

\section{PATIENTS AND METHODS \\ Patients}

This was a multicentre trial including six centres. Inclusion criteria were age 2-20 years, a diagnosis of SJIA, ${ }^{1}$ more than 6 months' disease duration, active systemic disease (disease-related fever and/ or C-reactive protein (CRP) $>20 \mathrm{mg} / \mathrm{l}$ and/or first hour erythrocyte sedimentation rate (ESR) $>20$ ) and significant overall disease activity at day 1 (D1) (at least three of the following criteria: (1) physician global assessment of disease activity $\geq 20 / 100$; (2) parent/patient assessment of disease effect on overall wellbeing $\geq 20 / 100$; (3) Childhood Health Assessment Questionnaire score $\geq 0.375 / 3$; (4) $\geq 2$ joints with active arthritis; (5) $\geq 2$ joints with nonirreversible limited range of motion and (6) ESR $\geq 30$ ) despite oral prednisone or prednisolone $\geq 0.3 \mathrm{mg} / \mathrm{kg}$ or $10 \mathrm{mg} /$ day (whichever was lower). Female subjects entering the study were prepubescent, sexually inactive or required to use effective contraception. Exclusion criteria included previous treatment with an IL-1 inhibitor or any condition contraindicating immunosuppressive treatment. Intravenous or intra-articular steroids, immunosuppressive drugs 
and disease-modifying antirheumatic drugs (DMARDs) had to be stopped at least 1 month before study onset or for longer periods of time depending on their half-life. All patients entering the study, and their parents for patients aged $<18$, gave written informed consent.

\section{Study design}

The study was approved by the local independent ethics committee and consisted of two parts (figure 1). Part 1 was a randomised, double-blind, placebo-controlled phase. At D1, eligible patients were randomised to receive either anakinra or placebo (1:1) from D1 to month 1 (M1) using a computer-generated random list. Patients were stratified by centres and randomisation was balanced across treatments and centres. The randomisation information included the randomisation number, the centre, the assigned treatment and the date of randomisation. Investigators, other caregivers, the patients and their parents remained blinded to the assigned treatment. The primary objective was to demonstrate a higher proportion of responders in group 1 than group 2 . No immunosuppressive drugs or DMARDs were allowed during the trial. Non-steroidal anti-inflammatory drugs and corticosteroids had to be taken at stable dosage for 1 month before D1 and until M1.

Part 2 was an open-label treatment period: all patients received anakinra after M1. Tapering the dose of corticosteroids was allowed after the M1 visit (reduction of $0.4-0.5 \mathrm{mg} / \mathrm{kg}$ monthly for daily doses of $\geq 1.5 \mathrm{mg} / \mathrm{kg}, 0.3-0.4 \mathrm{mg} / \mathrm{kg}$ for doses between 1 and $1.5 \mathrm{mg} / \mathrm{kg}, 0.2-0.3 \mathrm{mg} / \mathrm{kg}$ between 0.6 and $1 \mathrm{mg} / \mathrm{kg}, 0.1-0.2 \mathrm{mg} / \mathrm{kg}$ between 0.3 and $0.6 \mathrm{mg} / \mathrm{kg}, \leq 0.10 \mathrm{mg} / \mathrm{kg}$ for doses $<0.3 \mathrm{mg} / \mathrm{kg}$ ).

Measurement of serum amyloid A (SAA) and ferritin levels and the percentage of glycosylated ferritin were performed at D1, M1 and M6. Total ferritin concentration was measured on a Dimension RXL HM according to the guidelines of the manufacturer (Dade Behring, Paris, France). Glycosylated ferritin was determined according to the method of Worwood et al, ${ }^{11}$ with minor modifications as previously described. ${ }^{12}$ Pharmacokinetic (PK) analyses were performed on blood taken at M2 and M6. Concentrations of anakinra in plasma samples were determined using the antibody (Ab) ELISA purchased from R\&D Systems (Minneapolis, Minnesota, USA).

Patients who were naive from anti-pneumococcal immunisation received Pneumo23 immunisation at D1 in order to assess at M1 and M12 the effect of anakinra treatment on anti-pneumococcal $\mathrm{Ab}$ response to five capsular polysaccharides. ${ }^{13}$

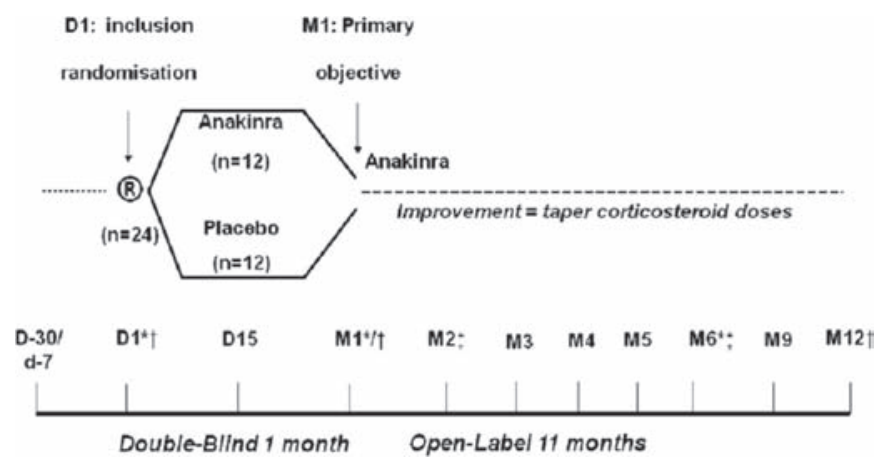

Figure 1 Study design. *Measurement of serum amyloid A and ferritin levels, assessment of the percentage of glycosylated ferritin, gene expression profiling analysis and cytokine measurements. tMeasurement of the concentration of anakinra in plasma (pharmacokinetic analyses). ‡Measurement of serum antipneumococcal antibodies. D, day; M, month.

\section{Gene expression profiling and cytokine measurements}

Blood samples were collected in Tempus tubes (ABI, Foster City, California, USA) from the patients at D1, M1 and M6 and from age and gender-matched healthy controls.

\section{Transcriptional module-based analysis}

This mining strategy has been described in detail elsewhere. ${ }^{14}$ In this study, RefSeq IDs were used to match probes between the Affymetrix U133 platform, which was used to generate the original set of modules using PBMC expression data, ${ }^{14}$ and Illumina Hu6 platform, which was used to hybridise whole blood samples from patients enrolled in this study and healthy controls. Unambiguous matches in Illumina were found for 2109 out of the 5348 Affymetrix probe sets. Illumina modules containing $<10$ genes were not included in the analysis.

\section{Cytokine multiplex analysis}

Serum samples were analysed using the cytokine assay kit (BioRad, Hercules, California, USA) according to the manufacturer's protocol.

\section{Assessment, outcome and safety measures}

The primary objective was to compare the efficacy after 1 month's treatment with anakinra ( $2 \mathrm{mg} / \mathrm{kg}$ subcutaneously daily, maximum $100 \mathrm{mg}$ ) or placebo in the two groups of patients. To be responders to a modified American College of Rheumatology Pediatric (ACRpedi) 30 score built for the purpose of the trial, patients had to fulfil the three following conditions: (1) ACRpedi 30 response $^{15}$; (2) absence of disease-related fever (body temperature $<38^{\circ} \mathrm{C}$ over the past 8 days) and (3) $50 \%$ decrease compared with D1 or normalisation of both CRP and ESR values.

Modified ACRpedi 30, 50, 70 and 100 responses, assessed throughout the study, included an improvement of $30 \%, 50 \%$, $70 \%$ or more and $100 \%$ respectively, in at least three of the six core criteria for juvenile rheumatoid arthritis and a worsening of 30 or more in no more than one of the criteria. The criteria were the physician's global assessment of disease activity and the patient's or the parents' global assessment of overall wellbeing, the number of joints with active arthritis, the number of joints with limited range of motion, the Childhood Health Assessment Questionnaire and ESR. ${ }^{15}$ A disease flare was defined as either (1) a reoccurrence of disease-related fever or any systemic symptom, or (2) an increase by twofold of either the ESR or CRP value, or (3) a worsening of $\geq 30 \%$ in at least three of the six ACRpedi core criteria and an improvement of $\geq 30 \%$ in no more than one of the criteria. If the number of joints with active arthritis was used as a criterion of flare and the patient initially had no active joints or only one active joint, an increase in the number of joints with active arthritis to at least two was required.

We also aimed to assess the number of patients who reached M6 with inactive disease, as defined by Wallace et a ${ }^{16}$ under a daily dose of predniso(lo)ne $<0.3 \mathrm{mg} / \mathrm{kg}$ or $10 \mathrm{mg}$, whichever was lower

Adverse events (AEs) were recorded throughout the study. Specific procedures were set up for serious AEs (SAEs). This trial was registered at http://www.clinicaltrials.gov (registration number: NCT00339157).

\section{Data analysis}

We expected at least $60 \%$ difference in the percentage of patients obtaining improvement in the anakinra-treated group (group 1) compared with the control group (group 2), with no 
more than $10 \%$ patients improving in group 2 . Given a $5 \%$ type I error, a 20\% type II error and a two-sided Fisher exact test, 12 patients per group were required. An intention-to-treat analysis was retained.

To explore whether each variable from the ACRpedi score, CRP, SAA and/or parent/patient assessment of pain were associated with response to treatment, the ratio (value at inclusion - value at M1)/value at inclusion, was compared in both groups. Qualitative and quantitative data were compared using Wilcoxon test and Fisher exact test, respectively. The $\mathrm{R}$ statistical software was used for statistical analysis.

\section{RESULTS}

\section{Demographic and baseline characteristics}

Twenty-seven patients were screened for entry into the study; 24 were eligible for randomisation (figure 2A). Demographic and disease characteristics are summarised in table 1.
Randomised, double-blind, placebo-controlled phase Response to the primary objective and assessment of individual variables

Eight of 12 patients (67\%) in group 1 and only 1 of $12(8 \%)$ in group 2 were responders at $\mathrm{M} 1(\mathrm{p}=0.003)$, therefore the primary objective of the trial was met.

Two patients from the control group stopped treatment after 5 and 11 days, respectively, owing to pain from injections and were withdrawn from the trial after the M1 visit; one of them, a child who presented a marked disease flare at D1, was the only responder at $\mathrm{M} 1$ in the control group.

There was a significant difference in favour of group 1 in the number of joints with active disease, physician general assessment of disease activity, CRP, ESR and SAA values at M1 compared with D1 (table 2).

A univariate analysis to explore whether any covariable might be associated with the response at $\mathrm{M} 1$ according to the treatment

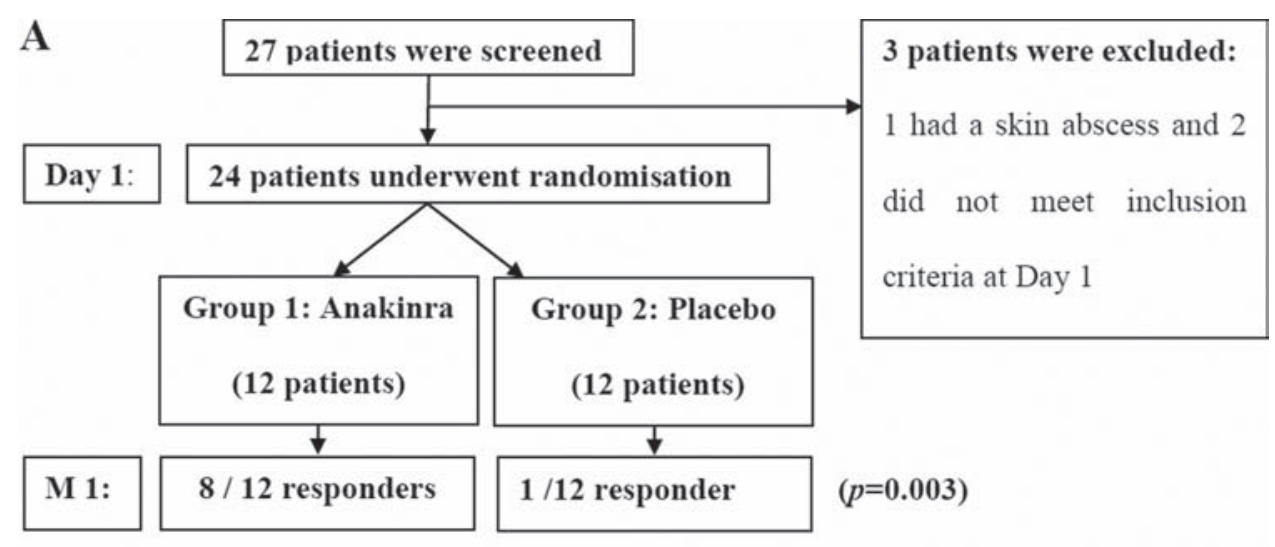

B Group 1 (Anakinra since Day 1)

Group 2 (Anakinra after Month 1)

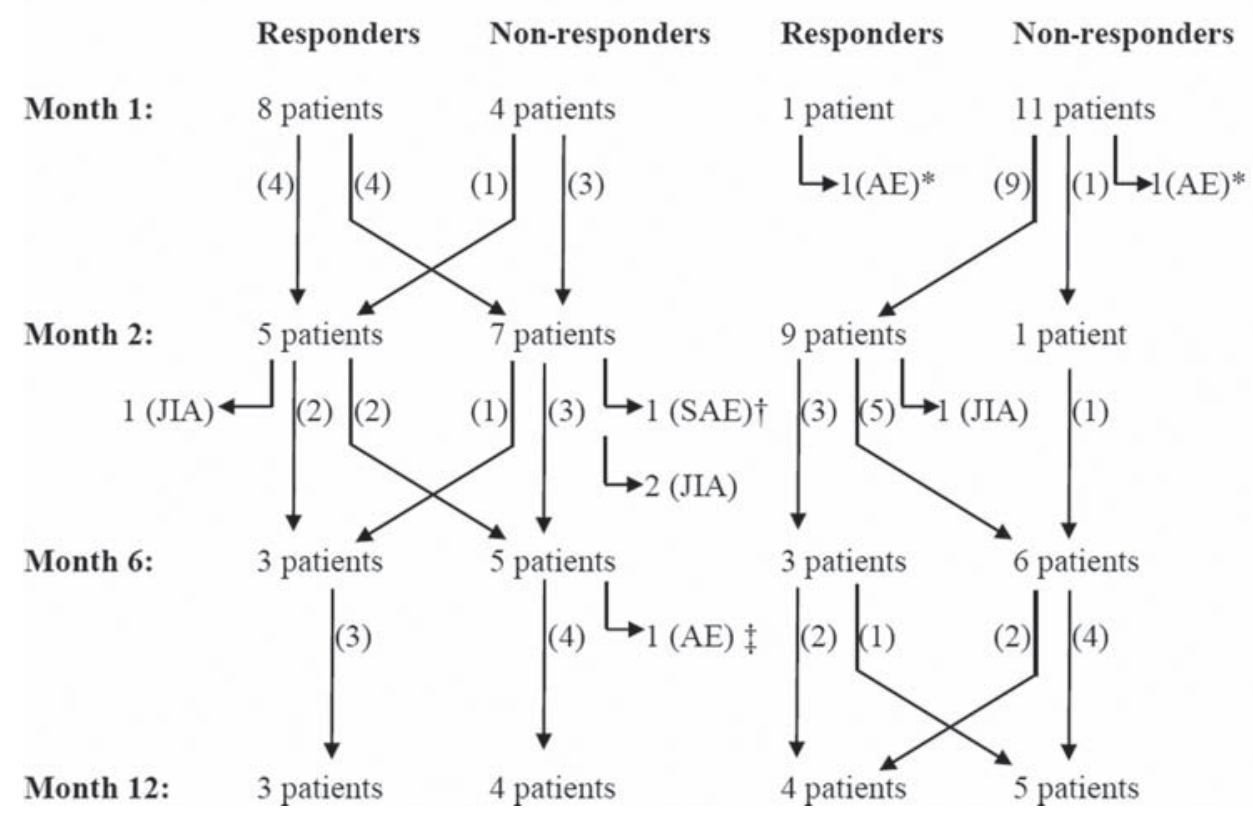

Figure 2 Patients' disposition. (A) Randomised placebo-controlled, double-blind trial (until M1). (B) Open-labelled phase (from M1 to M12). Arthritis activity leading to treatment withdrawal (= two patients withdrawn for a disease flare-up, at M2 and M3, respectively, and two patients withdrawn for a lack of response, at M4 and M5, respectively). * Two patients from the control group stopped treatment after 5 and 11 days, respectively, owing to pain from injections and were withdrawn from the trial after the M1 visit. †Cutaneous and digestive symptoms leading to the diagnosis of Crohn's disease shortly after M2. Inncrease of serum transaminases over five times the upper limit of normal at M6. AE, adverse event; JIA, juvenile idiopathic arthritis; $M$, month; SAE, serious adverse event. 
Table 1 Patients characteristics at study treatment onset

\begin{tabular}{|c|c|c|c|}
\hline Characteristics & Anakinra $(n=12)$ & Placebo $(n=12)$ & All patients $(n=24)$ \\
\hline \multicolumn{4}{|l|}{ Demographic features } \\
\hline Female, n (\%) & $7(58)$ & $8(67)$ & $15(63)$ \\
\hline Age, mean value, years $(S D)$ & $9.5(5.19)$ & $7.5(3.73)$ & $8.5(4.54)$ \\
\hline Disease mean duration, years (SD) & $4.2(3.33)$ & $3.2(1.95)$ & $3.7(2.73)$ \\
\hline \multicolumn{4}{|l|}{ Systemic features } \\
\hline Fever $\left(>38^{\circ} \mathrm{C}\right)$, no. of patients $(\%)$ & $4(33.3)$ & $5(41.7)$ & $9(37.5)$ \\
\hline 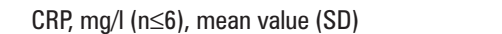 & $66(64.40)$ & $84(65.74)$ & $75(64.35)$ \\
\hline ESR first hour ( $n \leq 10)$, mean value (SD) & $44(23.37)$ & $57(27.85)$ & $50(25.89)$ \\
\hline $\mathrm{SAA}, \mathrm{mg} / \mathrm{l}(\mathrm{n} \leq 6.4)$, mean value (SD) & $366(262)$ & $368(229)$ & $367(241)$ \\
\hline High serum ferritin*, no. of patients & 2 & 3 & 5 \\
\hline \multicolumn{4}{|l|}{ Joint assessment } \\
\hline Active joints, mean no. (SD) & $16(13.12)$ & $16(15.84)$ & $16(14.23)$ \\
\hline Joints with LOM, mean no. (SD) & $16(14.88)$ & $17(14.91)$ & $17(14.57)$ \\
\hline \multicolumn{4}{|l|}{ Global assessments } \\
\hline Physician's VAS, mean value (SD) & $63(20.57)$ & $57(29.74)$ & $60(25.21)$ \\
\hline Parent's global VAS, mean value (SD) & $50(24.39)$ & $55(26.51)$ & $52(25.04)$ \\
\hline Parent's pain VAS, mean value (SD) & $50(25.73)$ & $53(25.89)$ & $51(25.28)$ \\
\hline $\mathrm{CHAQ}$, mean value (SD) & $1.67(0.845)$ & $1.44(0.625)$ & $1.55(0.736)$ \\
\hline \multicolumn{4}{|l|}{ Treatment with steroids (predniso(lo)ne) } \\
\hline Duration, mean, years (SD) & $3.9(2.93)$ & $2.7(2.10)$ & $3.3(2.56)$ \\
\hline Daily dose, mean, mg/kg (SD) & $0.52(0.237)$ & $0.66(0.373)$ & $0.59(0.313)$ \\
\hline \multicolumn{4}{|l|}{ Previous treatments with DMARDs, biological agents } \\
\hline DMARD and/or biological agent, no. of patients & 8 & 11 & 19 \\
\hline DMARD, no biological agent, no. of patients & 3 & 3 & 6 \\
\hline DMARD and biological agent, no. of patients & 5 & 8 & 13 \\
\hline Methotrexate, no. of patients & 8 & 11 & 19 \\
\hline Etanercept, no. of patients & 5 & 8 & 13 \\
\hline Others, no. of patients (no. of DMARDs) & $4\left(7^{\dagger}\right)$ & $4\left(6^{\ddagger}\right)$ & $8(13)$ \\
\hline \multicolumn{4}{|c|}{ 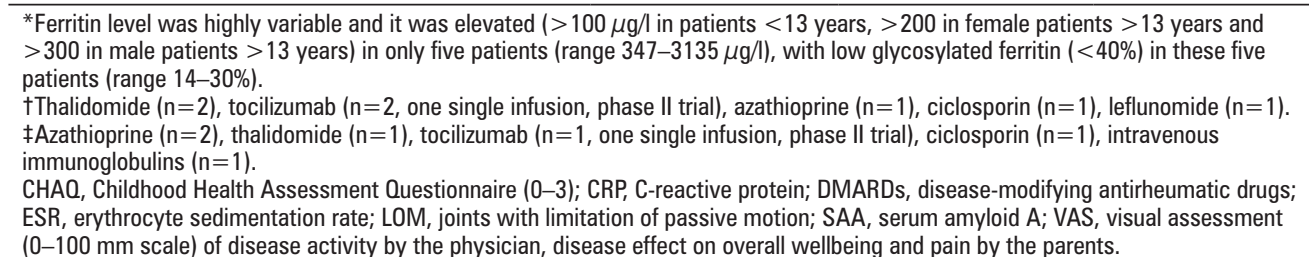 } \\
\hline
\end{tabular}

group failed to find any association. A multivariate analysis was not performed given the restricted number of patients.

\section{Safety and tolerability}

Fourteen AEs were recorded in group 1 and 13 in group 2 (table 3).There were no SAEs.

\section{Anti-pneumococcal antibody response}

At M1, the levels of postvaccination Abs against five pneumococcal capsular polysaccharide serotypes were not significantly different between the two groups. In group 1, 11 patients adequately responded to all serotypes and the 12th patient had a normal response to three of the five serotypes (online supplementary table S1).

\section{Gene expression profiling analyses}

Samples from 21 patients were included in these analyses, as blood from two patients was not properly collected and one patient was eventually diagnosed with Crohn's disease.

\section{Baseline SJIA blood gene expression profiling}

Analyses of blood from 21 patients with SJIA and 21 controls at D1 showed the differential expression of 986 transcripts (supplementary figure S1A and table S2). Among those, some of the most highly upregulated encode proteins involved in innate immunity activation, including (1) signalling components of the IL-1, IL-18 and Toll-like receptors (IL-1R2, IL-18RAP, IRAK3, TLR5, PGLYRP1), (2) inflammasome (CARD12/IPAF) and (3) anti-bacterial defence/inflammation (DEFA1, BPI, MPO ELA2,
CD163 and several members of the S100 family). Downregulated transcripts encode proteins involved in antigen presentation, lymphocyte lineage and signalling, as well as RNA processing/ splicing and DNA modification (supplementary figure S1B and table S2). Cytokine transcripts encoding IL1- $\beta$ and IL- 6 were not differentially expressed, but oncostatin (OSM) and the negative regulator of cytokine signalling 3 (SOCS3) transcripts were upregulated.

Changes in blood transcriptional signatures after 1 month At M1, 522 transcripts were significantly changed in patients treated with anakinra but not in the placebo-treated group (supplementary table S3). We found a significant upregulation of the purinergic receptor P2RX7, an ATP-gated ion channel involved in IL-1 $\beta$ processing and secretion, ${ }^{17}$ HLA-class II genes, the proteasome activator PSME2 and transcripts involved in interferon (IFN) signalling (SOCS1, STAT1 and STAT2). IL-1B was among the most significantly downregulated transcripts.

Module-level analysis of the SJIA transcriptional signature to follow response to anakinra treatment

A recently described analysis algorithm ${ }^{14}$ that permits comparison of the expression of 28 groups of transcriptionally coregulated genes (modules) in patients and controls was applied to the blood expression profiles (figure 3). At D1, patients from both groups displayed fairly homogeneous modular expression patterns with significant upregulation of innate immunity and underexpression of adaptive immunity modules. Modules 
including myeloid linage (M1.5 and M2.6), neutrophil (M2.2) and inflammation (M3.2 and 3.3) related transcripts were upregulated. Modules associated with erythrocytes (M2.3) and platelets (M1.2) were also upregulated. Conversely, modules containing transcripts related to lymphoid/T-cell lineage (M2.8 and M3.8), B cells (M1.3), T cells (M1.4) and cytotoxic cells (M2.1) were downregulated. At M1, several modular alterations were corrected in the anakinra-treated patients who responded to treatment (figure 3). Of the four non-responders, one patient was later diagnosed with Crohn's disease and blood from another patient was not properly collected; the two other patients did not show dysregulation of the modules at baseline and remained the same at M1. The modular alterations corrected in responders include transcripts within the platelet (M1.2), cytotoxic (M2.1) and erythropoiesis (M2.3) modules. None of these modules changed in the placebo group, therefore supporting the

Table 2 Responses at month 1

\begin{tabular}{|c|c|c|c|}
\hline \multirow[b]{3}{*}{ Response } & Group 1 & Group 2 & \multirow[b]{3}{*}{ p Value* } \\
\hline & Anakinra (n = 12) & Placebo ( $n=12$ ) & \\
\hline & \multicolumn{2}{|l|}{ Number of responders (\%) } & \\
\hline Primary objective (modified ACRpedi 30) $^{\dagger}$ & $8(67)$ & $1(8)$ & 0.003 \\
\hline Systemic symptoms responders ${ }^{\dagger}$ & $8(67)$ & $1(8)$ & 0.003 \\
\hline \multicolumn{4}{|l|}{ Primary objectives used in other trials } \\
\hline ACRpedi 30 responders & $11(92)$ & $7(58)$ & 0.059 \\
\hline ACRpedi 30 and no fever ${ }^{\ddagger}$ & $11(92)$ & $6(50)$ & 0.025 \\
\hline ACRpedi 30, no fever and CRP $<15 \mathrm{mg} / \mathrm{I}^{\S}$ & $10(83)$ & $3(25)$ & 0.004 \\
\hline \multicolumn{4}{|l|}{ Modified ACRpedi 50, 70 and 100 response $^{\dagger}$} \\
\hline Modified ACRpedi 50 responders & $7(58)$ & 0 & 0.005 \\
\hline Modified ACRpedi 70 responders & $5(42)$ & 0 & 0.038 \\
\hline Modified ACRpedi 100 responders & 0 & 0 & 1 \\
\hline Response to individual variables & \multicolumn{2}{|l|}{ Mean variation from D1 to M1 (\%) } & p Value ${ }^{\pi}$ \\
\hline CRP & -71 & -16 & 0.001 \\
\hline ESR & -64 & -18 & 0.002 \\
\hline SAA & -70 & -2 & $<0.001$ \\
\hline Number of active joints & -46 & -18 & 0.040 \\
\hline Number of joints with LOM & -36 & -20 & 0.148 \\
\hline CHAO & -37 & -9 & 0.236 \\
\hline Physician's disease activity assessment** & -63 & -20 & 0.002 \\
\hline Parent/patient's global assessment ${ }^{* *}$ & -36 & -23 & 0.544 \\
\hline Parent/patient's assessment of pain** & -29 & -21 & 0.219 \\
\hline \multicolumn{4}{|c|}{ 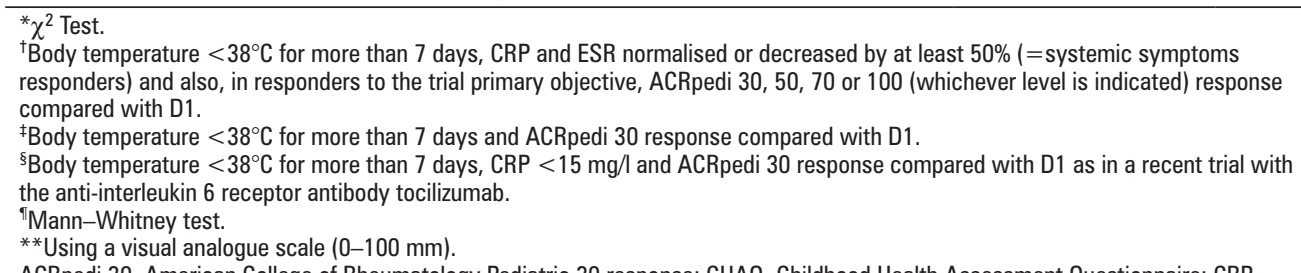 } \\
\hline
\end{tabular}

Table 3 Adverse events (AEs)

\begin{tabular}{|c|c|c|c|}
\hline \multirow[b]{3}{*}{ Adverse event } & \multicolumn{2}{|c|}{ Part A (double-blind) } & \multirow[t]{2}{*}{ Part 2 (M1-12) (open label) } \\
\hline & Group 1 & Group 2 & \\
\hline & Anakinra & Placebo & Anakinra \\
\hline Number of patients (patient-years ${ }^{*}$ ) & $12(1)$ & $12(1)$ & $22(15.17)$ \\
\hline Any $A E$, no. (/patient-year) ${ }^{\dagger}$ & $14(14)$ & $13(13)$ & $89(5.71)$ \\
\hline Serious $A E$, no. (/patient-year) & $0(0)$ & $0(0)$ & $5(0.33)^{\ddagger}$ \\
\hline Pain to injection, no. (/patient-year) & $8(8)$ & $6(6)$ & $15(0.99)$ \\
\hline Post-injection erythema, no. & 3 & 1 & $6(0.40)$ \\
\hline Infections, no. (/patient-year) & $2(2)$ & $2(2)$ & $44(2.90)$ \\
\hline ENT infections and laryngitis, no. & 1 & 1 & 20 \\
\hline Bronchitis, no. & 0 & 0 & 8 \\
\hline Gastroenteritis, no. & 1 & 1 & 3 \\
\hline Skin infections, no. & 0 & 0 & 4 \\
\hline Other infections, no. & 0 & 0 & $9^{\S}$ \\
\hline Vomiting, abdominal pain, no. & 0 & 1 & 9 \\
\hline Other $A E^{\mathbb{\pi}}$, no. (/patient-year) & $0(0)$ & $2(2)$ & $10(0.66)$ \\
\hline
\end{tabular}

\footnotetext{
*Patient-years = 12 patients in each group followed up for 1 month during the double-blind phase, 22 patients exposed to study treatment for a total of 182 months during the open-label phase (eight patients were withdrawn from the trial between M1 and M6).

${ }^{\dagger}$ Disease activity/flares was not systematically recorded as an AE.

‡Infections in four patients, vertebral collapse in one patient (these five patients continued the trial), skin and digestive symptoms leading to the diagnosis of Crohn's disease in one patient.

${ }^{\S}$ Varicella $(n=3)$, vulvar candidiasis $(n=2)$, isolated fever $(n=2)$, atypical pneumonitis, urinary tract infection. Favourable outcome in all cases, no patient withdrawn from the trial.

ISkin lesions $(n=5)$, haematuria $(n=2)$, back pain $(n=2)$, dental fracture, asthenia, vertigo.
} 

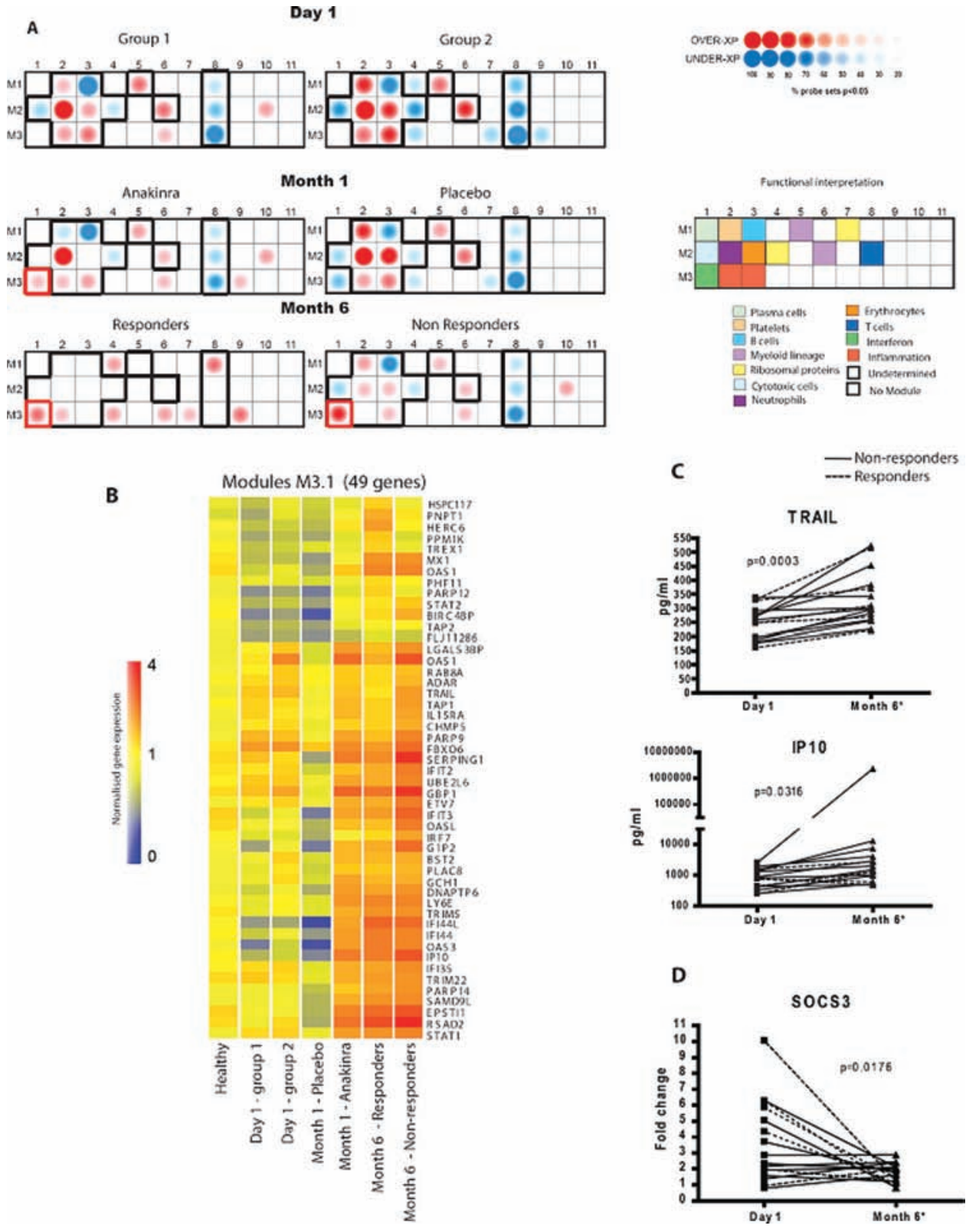

Figure 3 Modular analysis. (A) Annotated module map of patients with systemic-onset juvenile idiopathic arthritis (SJIA) at D1, M1 and M6. Expression levels in patients were compared with those of healthy controls. Spots indicate the proportion of genes that were significantly changed for each module (Mann-Whitney rank test, $\mathrm{p}<0.05$ ). The black outline indicates the core signature common in the two groups of patients at D1. (B) Forty-nine representative genes from the module 3.1 were rearranged by hierarchical clustering in order to reveal differential expression. Expression values are normalised for each gene to the healthy group. Transformed expression levels are indicated by colour scale, with red representing relatively high expression and blue indicating relatively low expression. (C) Levels of inducible proteins IP10 and TRAIL were measured in the serum of patients with SJIA at D1 and M6. All results were analysed using a paired t-test. (D) SOCS3 expression was measured in whole blood of patients by microarray at D1 and M6. Results were analysed using a paired t-test. D, day; M, month.

validity of the data at D1. We also observed the coordinated upregulation of type I IFN-inducible transcripts (M3.1) in the anakinra- but not in the placebo-treated group.

\section{Open-label phase}

Twenty-two patients entered the second phase of the study (figure 2B). Nine out of 10 patients from group 2 who switched to anakinra at M1 responded at M2. PK analyses revealed a trend towards lower anakinra concentrations at M2 and M6 in patients with lower weight and those who had failed to respond to anakinra after 1 month (residual anakinra concentration of $45.5 \pm 51 \mathrm{ng} /$ $\mathrm{ml}$ (range 20-122) vs $136.5 \pm 106 \mathrm{ng} / \mathrm{ml}$ (range 20-353) in responders), but overall this difference was not significant.
The dose of predniso(lo)ne had been reduced after the M1 visit in accordance with the protocol recommendations in all the responders who continued the trial (eight patients, all from group 1) and in seven other patients (four patients from group 1 and three patients from group 2) who had shown some improvement (investigator's decision). Three responders from group 1 in whom the dose of corticosteroids had been reduced were not responders at $\mathrm{M} 2$ anymore.

The dose of corticosteroids was then either maintained stable or reduced in accordance with the protocol recommendations at each visit (supplementary table S4).

Seventeen patients continued the trial until M6. Their mean daily dose of predniso(lo)ne was $0.18 \mathrm{mg} / \mathrm{kg}$ (median 0.16 , 
range $0-0.58)$; the six responders at $\mathrm{M} 6$ had a daily prednisone dose of $<10 \mathrm{mg}$ or $0.3 \mathrm{mg} / \mathrm{kg}$. These six patients, whose mean predniso(lo)ne dose at enrolment was $0.51 \mathrm{mg} / \mathrm{kg}$ (SD 0.32), were all responders to 1 month of anakinra treatment and had already achieved ACRpedi 50 or 70 improvement at this stage.

Sixteen patients reached M12; among seven responders, six had stopped corticosteroid treatment and five of them had inactive disease.

\section{Safety and tolerability}

Six patients developed a SAE. Four infections and one vertebral collapse had a favourable outcome and these five patients continued the trial; one patient was diagnosed with Crohn's disease shortly after M2 and withdrawn from the trial. This patient initially belong to group 1; he was a non-responder at M1; however, minor improvement had led the treating doctor to start tapering the dose of prednisone at this stage.

Other AEs mainly consisted of non-severe injection-site reactions and common infections (table 3). One patient stopped anakinra owing to a sudden increase in serum transaminases at M6.

\section{Anti-pneumococcal (Pneumo23) antibody response}

At M12, 12 patients were tested and an adequate Ab response was maintained in all cases (supplementary table S1).

\section{Changes in gene expression profiling at M6, modular analysis and correlation with clinical response to anakinra}

Expression of more than 500 transcripts changed significantly in the six patients who responded to anakinra at M6 (supplementary table S5).

The modular analysis of responders at $M 6(n=6)$ showed the normalisation of 11/13 original modular alterations, while non-responders still showed altered expression of 12 of them (figure 3A). Interestingly, overexpression of type I IFN-inducible genes (module 3.1) was observed in every group after initiation of anakinra treatment (both at $\mathrm{M} 1$ and $\mathrm{M} 6$ ) regardless of the clinical response (figure $3 \mathrm{~B}$ ). To support the validity of the transcriptional data, we measured the protein levels of two IFNinducible proteins (IP10 and TRAIL) and found that they were significantly increased in the serum of anakinra-treated patients at M6 (figure 3C). Accordingly, SOCS3 expression, which could explain the cross-regulation of IL- $1 \beta$ and type I IFN, was downregulated in the majority of patients at $\mathrm{M} 6$ (figure $3 \mathrm{D}$ ).

\section{DISCUSSION}

This double-blind, placebo-controlled study demonstrated the efficacy of anakinra in treating corticosteroid-dependent patients with SJIA, as a significantly higher proportion of responders was observed after 1 month of treatment compared with placebo. However, a loss of response was observed in most patients over time.

The efficacy of anakinra treatment in SJIA, at least in the short term, concorded with previous reports. ${ }^{6-10}$ However, the only other placebo-controlled trial testing anakinra in JIA showed no significant improvement versus placebo, ${ }^{18}$ illustrating that marked response to IL- 1 inhibition is not characteristic of all JIA subtypes. The lack of sustained response in several patients may have been related to several factors. First, most patients had diffuse polyarthritis at enrolment but no fever; as previously reported, ${ }^{9}$ anakinra seemed less effective on the arthritis than on systemic features. Second, PK data suggested that low-weight children might have benefited from a higher anakinra dosage. Third, we included patients with active SJIA, steroid-dependency and a minimal disease duration of 6 months, therefore difficult to treat ${ }^{5}$; loss of responses may have been favoured by the study design that precluded the use of associated DMARDs and allowed tapering of the corticosteroid dose at an early stage, a strategy designed to minimise the risk of treatment-related complications.

This study has limitations owing to the small number of patients and the short duration of the double-blind phase. In particular, we were unable to properly assess which patients' characteristics might predict response to treatment. In recent reports, the absence of small joint involvement, a smaller number of joints with active disease and an increased absolute neutrophil count were reported to be predictive of response to anakinra. 910

No severe infections occurred in this trial and anti-pneumococcal $\mathrm{Ab}$ response was not affected by anakinra treatment. However, severe infections have been reported under anakinra in adults with rheumatoid arthritis. ${ }^{19}$ One patient here was diagnosed with inflammatory bowel disease, a complication reported in some patients initially diagnosed with JIA and treated with anti-TNF agents. ${ }^{3} 2021$ An additional patient experienced a sudden rise in serum transaminases. In order to draw definitive conclusions about the safety of anakinra in SJIA, further studies with larger numbers of patients and longer follow-up need to be implemented.

As expected, the ex vivo genomic analysis of patient blood at D1 showed upregulation of transcripts related to innate immunity and haematopoiesis (supplementary table S2). Although several hereditary and sporadic IL- $1 \beta$-mediated diseases involve alterations in NLRP3 inflammasome components, ${ }^{22}$ no altered expression of NLRP3 inflammasome-related transcripts was observed. However, two different flagellin receptors (CARD12 and TLR5) were significantly upregulated in the majority of patients at D1 (supplementary table S2) and normalised in the responder group at M6 (supplementary table S5). Whether the triggering of flagellin receptors is involved in the pathogenesis of SJIA remains to be elucidated. Alterations in the blood gene expression profiles of patients with SJIA at the time of randomisation were highly reproducible in the treatment and placebo groups. This was best visualised using a strategy based on the analysis of modules composed of transcriptionally co-regulated genes. ${ }^{14}$ Longitudinal changes correlating with disease resolution were found upon follow-up. Changes probably induced by treatment, regardless of clinical response, were also identified. Strikingly, an upregulation of type I IFNregulated transcripts, which is not a feature of untreated SJIA, ${ }^{7}$ 23-25 was induced in the majority of anakinra-treated patients at M1 and M6. Accordingly, two IFN-inducible proteins were also significantly upregulated in the serum samples of patients at M6. The molecular and cellular basis for an IL-1 $\beta$ /type I IFN cross-regulation in SJIA remains unknown. Inhibition of type I IFN signalling by IL-1 $\beta$ might occur through the induction of SOCS3, which is a negative regulator of IL-1-dependent acute inflammatory arthritis and osteoclast generation. ${ }^{26} 27$ Conversely, type I IFN decreases IL-1 $\beta$ and induces IL-1Ra production by PBMCs in vitro. ${ }^{28} \mathrm{~A}$ similar cross-regulation has been described for type I IFN and TNF $\alpha$. Thus, patients with SJIA treated with TNF antagonists develop a blood IFN signature. ${ }^{29}$ Furthermore, a significant proportion of patients with rheumatoid arthritis and Crohn's disease treated with TNF antagonists develop anti-nuclear Abs and some of them display symptoms of systemic lupus erythematosus, a type I IFN-mediated disease. ${ }^{29}$ Whether the upregulation of type I IFN-inducible genes in anakinra-treated patients could have clinical consequences through the triggering of adaptive immunity remains to be determined. 
In conclusion, a significant proportion of patients with SJIA responded to IL-1 blockade as dramatically as patients with autoinflammatory syndromes, confirming a key role for IL-1 in a wide spectrum of diseases. ${ }^{30}$ Furthermore, clinical response and cytokine cascades induced upon IL1 blockade can be followed using blood gene expression profiling.

Acknowledgements Professor Kone-Paut, Drs Lemelle, Fouillet-Desjonqueres, Florkin and Neven were co-investigators. The Centres d'Investigation Clinique of Necker-Enfants Malades, Paris (Pr Bresson), Lyon (Dr Kassai, Ms Masson), Bordeaux (Ms Vautrat and Nacka), and Ms Marie, clinical research nurse from Bicetre took an active part in the trial. Drs Broissand, Pivot, Ghezzoul, Prevot, Taburet and Sterlingot were involved as pharmacists, Dr Urien in PK analyses, Ms Menoni and Sotou-Bere in data management and Karolina Palucka in rewriting important sections of the paper.

Funding The promoter was the Institut Francais pour la Recherche Scientifique et Medicale (INSERM). Financial support was obtained from AMGEN (which had no role in analysis and reporting phase), INSERM, the French Society for Inflammatory Diseases in Pediatric Rheumatology (SOFREMIP), the Association for the Development of Pediatric Rheumatology (ADRI) and the French association for patients with Juvenile Arthritis KOURIR.

\section{Competing interests None.}

Contributors All authors were involved in drafting of the article or revising it critically for important intellectual content, and all authors approved the final version to be published. PQ had full access to all the data in the study and takes responsibility for the integrity of the data and the accuracy of the data analysis. Study conception and design: $\mathrm{PQ}$ and $\mathrm{PL}$; acquisition of data: $\mathrm{PQ}, \mathrm{FA}, \mathrm{RC}, \mathrm{PP}, \mathrm{AD}$ and $\mathrm{OR}$; analysis and interpretation of data: $P Q, F A, C M, C B, X B, A B, J B, D C, J-M T, J B$, PL and VP.

Ethics approval This study was conducted with the approval of the Necker-Enfants Malades Hospital, Paris, France, Commite pour la protection des personnes.

Provenance and peer review Not commissioned; externally peer reviewed.

\section{REFERENCES}

1. Petty RE, Southwood TR, Manners P, et al. International League of Associations for Rheumatology classification of juvenile idiopathic arthritis: second revision, Edmonton, 2001. J Rheumatol 2004;31:390-2.

2. Woo P, Southwood TR, Prieur AM, et al. Randomized, placebo-controlled, crossover trial of low-dose oral methotrexate in children with extended oligoarticular or systemic arthritis. Arthritis Rheum 2000;43:1849-57.

3. Quartier $\mathbf{P}$, Taupin P, Bourdeaut $\mathrm{F}$, et al. Efficacy of etanercept for the treatment of juvenile idiopathic arthritis according to the onset type. Arthritis Rheum 2003;48:1093-101.

4. Yokota S, Imagawa T, Mori M, et al. Efficacy and safety of tocilizumab in patients with systemic-onset juvenile idiopathic arthritis: a randomised, double-blind, placebo-controlled, withdrawal phase III trial. Lancet 2008;371:998-1006.

5. Spiegel LR, Schneider R, Lang BA, et al. Early predictors of poor functional outcome in systemic-onset juvenile rheumatoid arthritis: a multicenter cohort study. Arthritis Rheum 2000;43:2402-9.

6. Pascual V, Allantaz F, Arce E, et al. Role of interleukin-1 (IL-1) in the pathogenesis of systemic onset juvenile idiopathic arthritis and clinical response to IL-1 blockade. J Exp Med 2005;201:1479-86.

7. Allantaz F, Chaussabel D, Stichweh D, et al. Blood leukocyte microarrays to diagnose systemic onset juvenile idiopathic arthritis and follow the response to IL-1 blockade. $J$ Exp Med 2007;204:2131-44

8. Lequerré T, Quartier P, Rosellini D, et al. Interleukin-1 receptor antagonist (anakinra) treatment in patients with systemic-onset juvenile idiopathic arthritis or adult onset Still disease: preliminary experience in France. Ann Rheum Dis 2008;67:302-8.
9. Gattorno M, Piccini A, Lasigliè D, et al. The pattern of response to anti-interleukin-1 treatment distinguishes two subsets of patients with systemic-onset juvenile idiopathic arthritis. Arthritis Rheum 2008:58:1505-15.

10. Zeft A, Hollister R, LaFleur B, et al. Anakinra for systemic juvenile arthritis: the Rocky Mountain experience. J Clin Rheumatol 2009;15:161-4.

11. Worwood M, Cragg SJ, Wagstaff M, et al. Binding of human serum ferritin to concanavalin A. Clin Sci 1979;56:83-7.

12. Vignes S, Le Moël G, Fautrel B, et al. Percentage of glycosylated serum ferritin remains low throughout the course of adult onset Still's disease. Ann Rheum Dis 2000;59:347-50.

13. Jeurissen A, Moens L, Raes M, et al. Laboratory diagnosis of specific antibody deficiency to pneumococcal capsular polysaccharide antigens. Clin Chem 2007:53:505-10.

14. Chaussabel D, Quinn C, Shen J, et al. A modular analysis framework for blood genomics studies: application to systemic lupus erythematosus. Immunity 2008;29:150-64.

15. Giannini EH, Ruperto N, Ravelli A, et al. Preliminary definition of improvement in juvenile arthritis. Arthritis Rheum 1997:40:1202-9.

16. Wallace CA, Ruperto N, Giannini E. Preliminary criteria for clinical remission for select categories of juvenile idiopathic arthritis. J Rheumatol 2004;31:2290-4.

17. Solle M, Labasi J, Perregaux DG, et al. Altered cytokine production in mice lacking P2X(7) receptors. J Biol Chem 2001;276:125-32.

18. Ilowite $\mathbf{N}$, Porras 0 , Reiff A, et al. Anakinra in the treatment of polyarticular-course juvenile rheumatoid arthritis: safety and preliminary efficacy results of a randomized multicenter study. Clin Rheumatol 2009;28:129-37.

19. Salliot C, Dougados M, Gossec L. Risk of serious infections during rituximab, abatacept and anakinra treatments for rheumatoid arthritis: meta-analyses of randomised placebo-controlled trials. Ann Rheum Dis 2009;68:25-32.

20. Ruemmele FM, Prieur AM, Talbotec C, et al. Development of Crohn disease during anti-TNF-alpha therapy in a child with juvenile idiopathic arthritis. J Pediatr Gastroenterol Nutr 2004;39:203-6.

21. Dallocchio A, Canioni D, Ruemmele F, et al. Occurrence of inflammatory bowel disease during treatment of juvenile idiopathic arthritis with etanercept: a French retrospective study. Rheumatology (Oxford) 2010;49:1694-8.

22. Martinon F, Mayor A, Tschopp J. The inflammasomes: guardians of the body. Annu Rev Immunol 2009;27:229-65.

23. Fall N, Barnes M, Thornton S, et al. Gene expression profiling of peripheral blood from patients with untreated new-onset systemic juvenile idiopathic arthritis reveals molecular heterogeneity that may predict macrophage activation syndrome. Arthritis Rheum 2007:56:3793-804.

24. Palucka AK, Blanck JP, Bennett L, et al. Cross-regulation of TNF and IFN-alpha in autoimmune diseases. Proc Natl Acad Sci USA 2005;102:3372-7.

25. Ogilvie EM, Khan A, Hubank $M$, et al. Specific gene expression profiles in systemic juvenile idiopathic arthritis. Arthritis Rheum 2007;56:1954-65.

26. Wong PK, Egan PJ, Croker BA, et al. SOCS-3 negatively regulates innate and adaptive immune mechanisms in acute IL-1-dependent inflammatory arthritis. J Clin Invest 2006;116:1571-81.

27. Pauli EK, Schmolke M, Wolff T, et al. Influenza A virus inhibits type I IFN signaling via NF-kappaB-dependent induction of SOCS-3 expression. PLoS Pathog 2008; 4:e1000196

28. Martinelli S, Urosevic M, Daryadel A, et al. Induction of genes mediating interferondependent extracellular trap formation during neutrophil differentiation. J Biol Chem 2004;279:44123-32.

29. Pascual V, Farkas L, Banchereau J. Systemic lupus erythematosus: all roads lead to type I interferons. Curr Opin Immunol 2006;18:676-82.

30. Dinarello CA. Interleukin-1 beta and the autoinflammatory diseases. N Engl J Med 2009;360:2467-70. 